\title{
Effect of Tapentadol on Experimental Model of Orofacial Pain - a Pilot Study
}

\author{
Jitka FRICOVÁ ${ }^{2}$, Helena LAINCZOVÁ ${ }^{2}$, Jan NEDVÍDEK ${ }^{1}$, Richard ROKYTA ${ }^{1}$ \\ ${ }^{1}$ Department of Physiology, Third Faculty of Medicine, Charles University, Prague, Czech \\ Republic, ${ }^{2}$ Department of Anaesthesiology, Resuscitation and Intensive Care, Pain Management \\ Center, First Faculty of Medicine, Charles University, Prague, Czech Republic
}

Received March 30, 2020

Accepted October 16, 2020

\begin{abstract}
Summary
Acute orofacial pain is associated with significant disability and has a detrimental impact on quality of life. Although various origins of the pain in trigeminal territory can be identified an odontogenic pathology is the most common cause of acute orofacial pain in patients. Due to complex pathophysiology drugs with multitarget action might provide beneficial effect in pain management. The aim of the present study was to experimentally examine the anti-nociceptive effects of tapentadol, an opioid agonist and a norepinephrine reuptake inhibitor (MOR/NRI), in our animal model of orofacial pain. We tested the effect of tapentadol at gradual doses of 1,2 and $5 \mathrm{mg} / \mathrm{kg}$ during thermal and mechanical stimulation in the trigeminal area of adult rats. We observed that tapentadol exhibits antinociceptive effect at dosages of $2 \mathrm{mg} / \mathrm{kg}$ and $5 \mathrm{mg} / \mathrm{kg}$ and only in association with mechanical stimulation.
\end{abstract}

\section{Key words}

Orofacial pain - Experimental orofacial model - Opioids • Tapentadol • Mechanical and thermal stimulation • Nociception

\section{Corresponding author}

J. Fricová, Department of Anaesthesiology, Resuscitation and Intensive Care, Pain Management Center, First Faculty of Medicine, Charles University, Karlovo Náměstí 32, 12808 Prague 2, Czech Republic. E-mail: j.fricova@seznam.cz

\section{Introduction}

Orofacial pain, a pain originating from the territory of trigeminal nerve, is a significant cause of disability and decline in quality of life. Odontogenic pathologies represent most common sources of acute orofacial pain. Chronic orofacial pain may be unilateral or bilateral with a persistent or seizure course. Recent research expects the involvement of the peripheral and central nervous systems as a mechanism in pathophysiology of orofacial pain (Fricová et al. 2019). An analgesic drug Tapentadol has dual synergistic agonistic effects (MOR/NRI) on $\mu$ opioid receptors (MOR) and noradrenaline reuptake inhibition (NRI). Tapentadol also activates alpha-2 receptors in the spinal cord (Bee et al. 2011). This mechanism may be responsible for its very good analgesic effect on neuropathic pain. Several III. phase studies have produced favorable results. Reports from approximately 4000 followed patients with severe chronic lower back pain and osteoarthritis treated with tapentadol (at doses of 100-250 mg twice a day (BID) have found the same positive analgesic effect as patients treated with oxycodone 20-50 mg BID. During 12 weeks of therapy, patients treated with tapentadol reported significantly lower incidence of side effects with the maintained analgesic effects (Buynak et al. 2010, Steigerwald et al. 2012). The pharmacological profile of tapentadol is also positive as it does not have any active metabolites, has low plasma protein binding, and does not depend on cytochrome P450 (CYP) metabolism. Tapentadol develops tolerance relatively slowly in comparison with other opioids. Potential antidepressant and anxiolytic effects described in clinical experience by numerous physicians are also very interesting, although, this feature 
has not been the subject of studies yet. Diabetic polyneuropathy (DNP) is a new indication of neuropathic pain for tapentadol, which has shown a very good effect compared to placebo. It was administered in doses of 100 up to $250 \mathrm{mg}$ BID and was compared to placebo in patients with moderate to severe pain as a result of chronic DNP. At the onset of therapy in patients with DPN, pain relief was assessed after three weeks of treatment when a titration was performed. This study (NCT01041859), which was done only in the U.S., showed the safety, effectiveness, and good tolerance of tapentadol. Clinical assessments were sufficient for FDA (Food and Drug Agency) approval in April 2012 and for the treatment of neuropathic pain associated with DNP. The use of tapentadol in combination with other antineuropathic medication also works well in patients with chronic neuropathic pain (Fricová 2014, Hakl 2015). The most frequent diagnoses in which tapentadol holds potential benefits are fibromyalgia, post-amputation pain, the neuropathic pain component in patients suffering from chronic lower back pain, and in various tumor pain syndromes. In our previous investigation (Fricova et al. 2018) we used the application of tapentadol in doses of 1 and $2 \mathrm{mg} / \mathrm{kg}$ in rats and we proved that the antinociceptive effect of tapentadol is realized only at the dosage of $2 \mathrm{mg}$ per kilogram of tapentadol.

In present study, we have used the experimental model of orofacial pain (UGO BASILE), which measure hypersensitivity to thermal or mechanical stimulation in the trigeminal area in rats.

A total of 42 adult male Wistar rats (aged of 8-10 weeks), weighing 300-400 g were used in the experiment (Velaz/Charles River). The effect of
Tapentadol on the frequency and duration of stimulation intervals was measured. A dose of saline or tapentadol in the same volume was administered i.p. to rats $60 \mathrm{~min}$ before being admitted to the apparatus. We tested the effect of tapentadol at gradual doses $1 \mathrm{mg} / \mathrm{kg}$ (10 rats), $2 \mathrm{mg} / \mathrm{kg}$ (10 rats) and $5 \mathrm{mg} / \mathrm{kg}$ (22 rats). The control group received $0.1 \mathrm{ml} / 100 \mathrm{~g}$ saline intraperitoneally. The experiments were performed under the approval of the Ethics Committee at the Third Faculty of Medicine (Ethics Committee No. MSMT-273/2017-2, 6.1.2017), Charles University and are in agreement with the Czech Government Requirements under the Policy of Humans Care of Laboratory Animals (No. 86/609/EEC) and with the subsequent regulations of the Ministry of Agriculture of the Czech Republic. To examine the anti-nociceptive effects of tapentadol an orofacial stimulation apparatus developed by Ugo Basile was used in these experiments (Fig. 1). This device is designed to mechanically or thermally stimulate the trigeminal region and measure its length of stay of the subject in the area of stimulation. During the experiment, the subject was offered a reward, for which he must visit the area of stimulation (which is discomfort for him). The monitored factor is the time for which the subject is willing to endure this discomfort. One measurement cycle was limited to $10 \mathrm{~min}$. Measurements were repeated individually and then statistically evaluated. For the overall comparison of the saline and tapentadol groups, a $t$-test was used to judge the effect of tapentadol. In the absence of a normal distribution of data, the Mann-Whitney Rank Sum Test or Wilcoxon Signed Rank Test was used. The results are presented in the form of a percentage change after administration of tapentadol as mean \pm SEM.
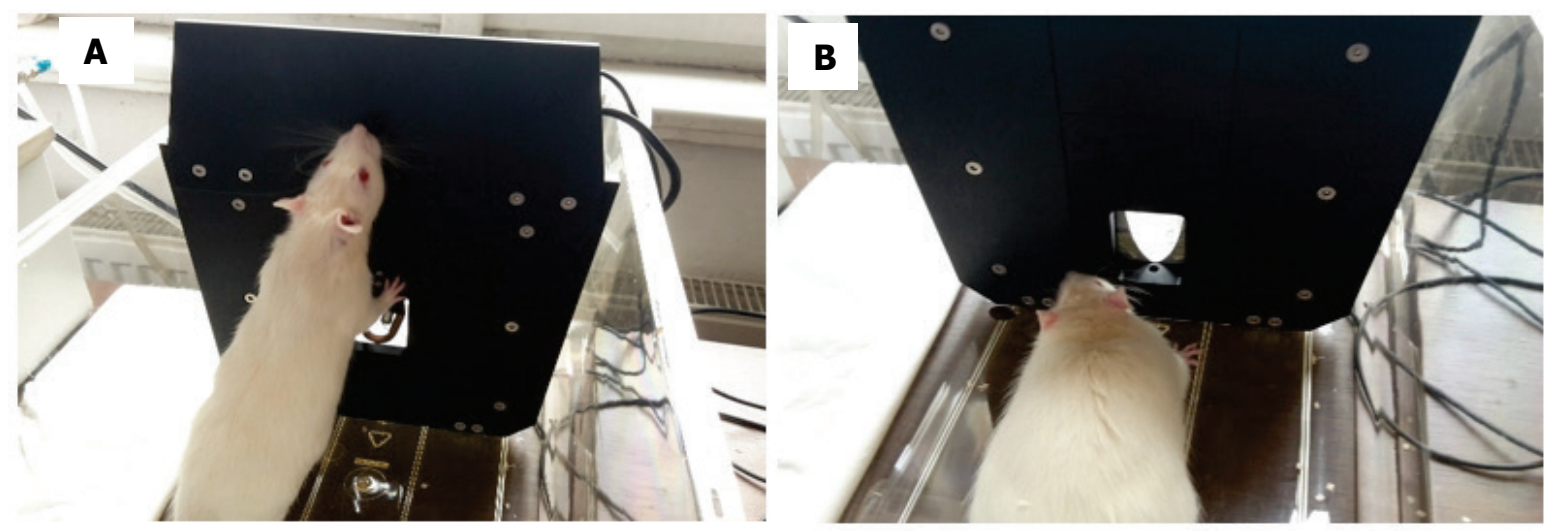

Fig. 1. The Orofacial Stimulation Test, developed by Ugo Basile, measures hypersensitivity to thermal or mechanical stimulation of the trigeminal area. In the experiment, rats voluntarily contact a thermal (A) or a mechanical (B) stimulator with their unshaved vibrissal pad in order to access a food reward. Data regarding the duration of feeding and the number of feeding attempts were obtained; attempts were determined based on the interruption of an infrared barrier traversing the opening to the reward. Feeding time and number of attempts were found to be highly dependent on changes in the applied thermal or mechanical stimulus. 
$1 \mathrm{mg}$ per kilogram of tapentadol has no effect on the thermal and mechanical stimulation. Tapentadol at both doses $2 \mathrm{mg} / \mathrm{kg}$ and $5 \mathrm{mg} / \mathrm{kg}$ increased tolerance (decreased sensitivity) to mechanical stimulation (Mann-Whitney Rank Sum Test, $\mathrm{P}<0.05)$. In the case of the dose of $5 \mathrm{mg} / \mathrm{kg}$ there was also a slight decrease in the frequency of visits of the water chamber (Fig. 2A, Fig. 3A). Tapentadol at both doses $2 \mathrm{mg} / \mathrm{kg}$ and $5 \mathrm{mg} / \mathrm{kg}$ decreased the tolerance (increased sensitivity) to thermal stimulation (Mann-Whitney Rank Sum Test, $\mathrm{P}<0.001$ ). In the case of the dose of $5 \mathrm{mg} / \mathrm{kg}$ at the same time, the frequency of visits of the water chamber increased (Fig. 2B, Fig. 3B).
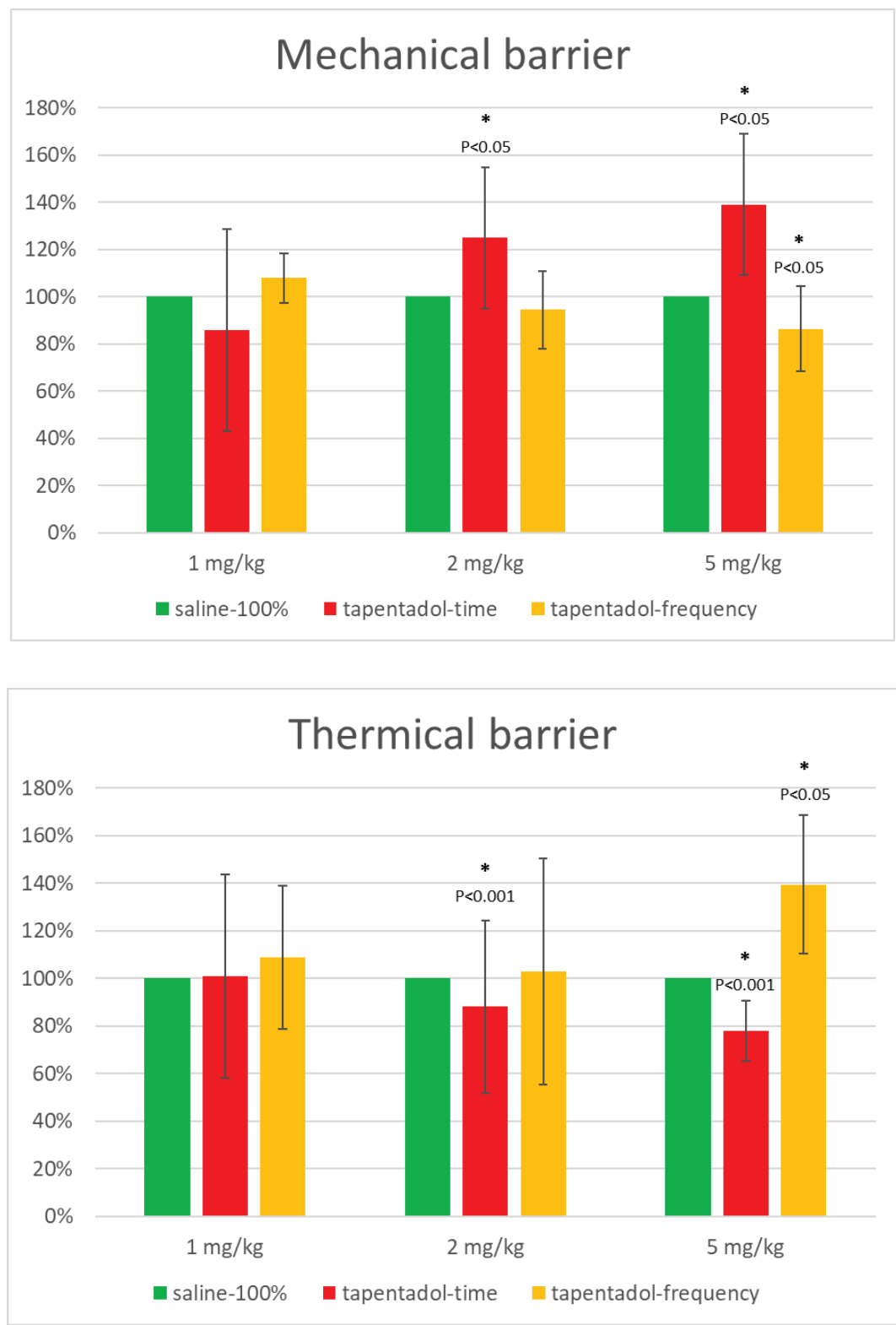

Fig. 2A. The relative difference in length of stay and frequency of visits after administration of saline resp. tapentadol in a water chamber with a mechanical entry barrier. GREEN bars are controls, RED bars are length of stay, YELLOW bars are frequency of visits.

Fig. 2B. The relative difference in length of stay and frequency of visits after administration of saline resp. tapentadol in a water chamber with a thermal barrier. GREEN bars are controls, RED bars are length of stay, YELLOW bars are frequency of visits.
We have demonstrated that anti-nociceptive effect of tapentadol is realized at doses of 2 and $5 \mathrm{mg} / \mathrm{kg}$ in association with mechanical stimulation. Although intraperitoneal administration of tapentadol $(2 \mathrm{mg} / \mathrm{kg})$ increased mechanical antinociception in rats application of higher dose of tapentadol $(5 \mathrm{mg} / \mathrm{kg})$ additionally affects also thermal stimulation and prolongs the duration of drinking.

Tapentadol has a dual mechanism of action, namely it is an agonist on $\mu$ opioid receptors and noradrenaline reuptake inhibitor (Fig. 4). In addition, it has been shown that tapentadol also activates alpha- 2 receptors in the spinal cord (Bee et al. 2011). Our data indicates that thermal threshold testing is not an ideal model for evaluating the antinociceptive effects of drugs with a dual mechanism of action. However, tramadol has a similar mechanism of action as tapentadol, and oral administration of tramadol produces thermal anti-nociception in a dose dependent manner (Siao and Ilkiw 2009). 

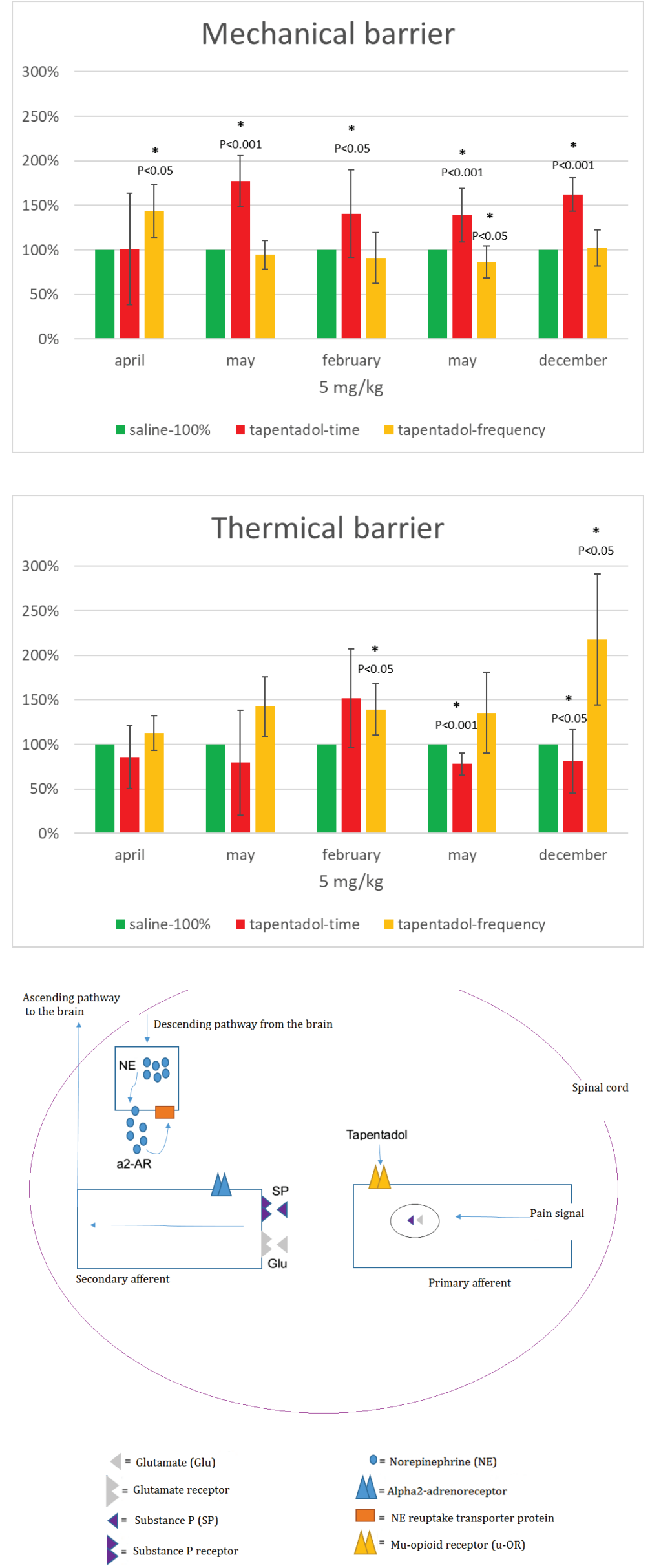

Fig. 3A. The relative difference in length of stay and frequency of visits after administration of saline resp. tapentadol in a water chamber with a mechanical entry barrier. GREEN bars are controls, RED bars are length of stay, YELLOW bars are frequency of visits. After the tapentadol dose, the time spent in the chamber is longer.

Fig. 3B. The relative difference in length of stay and frequency of visits after administration of saline resp. tapentadol in a water chamber with a thermal barrier to entry. GREEN bars are controls, RED bars are length of stay, YELLOW bars are frequency of visits.

Fig. 4. Dual mechanism of tapentadol (adapted from Tzschentke et al. 2007). 
Tapentadol was injected into laboratory rats and its effect on food rewards was observed. The right central nucleus of the amygdala $(\mathrm{CeA})$ is a critical part of the lateral spinal pain pathway, regulates descending pain pathways, and is key in the emotional-affective components of pain. Tapentadol has a dual mechanism of action; some support the norepinephrine reuptake inhibition pathway as being primarily responsible for it analgesic effects. Thus, it is possible that thermal threshold testing is not an ideal model for evaluating the antinociceptive effects of drugs with a dual mechanism of action. However, tramadol has a similar mechanism of action as tapentadol, and oral administration of tramadol produces thermal anti-nociception in a dose dependent manner (Siao and Ilkiw 2009). Nonetheless, it is clear that clinical pain is complex and that more than one type of nociceptive stimulus (Doodnaught et al. 2018). Further studies has to be conducted to elucidate this interesting discrepancy in obtained data. In conclusion, we have shown orofacial stimulation test UGO Basile a reliable approach for monitoring antinociceptive effects of drugs with potential analgesic action.

\section{Conflict of Interest}

There is no conflict of interest.

\section{Acknowledgements}

The work was supported by the grant of Charles University PROGRES Q 35.

\section{References}

BEE LA, BANNISTER K, RAHMAN W, DICKENSON AH: Mu-opioid and noradrenergic alpha(2)- adrenoceptor contributions to the effects of tapentadol on spinal electrophysiological measures of nociception in nerveinjured rats. Pain 152: 131-139, 2011. https://doi.org/10.1016/i.pain.2010.10.004

BUYNAK R, SHAPIRO DY, OKAMOTO A, VAN HOVE I, RAUSCHKOLB C, STEUP A, LANGE B, LANGE C, ETROPOLSKI M: Efficacy and safety of tapentadol extended release for the management of chronic low back pain: results of a prospective, randomized, double-blind, placebo-and active-controlled Phase III study. Expert Opin Pharmacother 11: 1787-1804, 2010. https://doi.org/10.1517/14656566.2010.497720

DOODNAUGHT GM, MONTEIRO B, EDGE D, STEAGALL PV: Thermal antinociception after buccal administration of a high-concentration formulation of buprenorphine (Simbadol) at $0.24 \mathrm{mg} \mathrm{kg}-1$ in conscious cats. Vet Anaesth Analg 45: 714-716, 2018. https://doi.org/10.1016/j.vaa.2018.05.007

FRICOVÁ J: Tapentadol v léčbě chronické nenádorové bolesti, nové účinné analgetikum pro léčbu diabetické polyneuropatie? (Article in Czech) Bolest 17: 107-111, 2014.

FRICOVÁ J, ENGLEROVÁ K, NEDVÍDEK J, ROKYTA R: The treatment of orofacial pain by using transcranial direct current stimulation. Physiol Res 68 (Suppl 3): S367-S372, 2019. https://doi.org/10.33549/physiolres.934361

HAKL M: Novinky ve farmakoterapii bolesti. (Article in Czech) Med Praxi 12: 19-21, 2015.

FRICOVA J, ROKYTA R, NEDVIDEK J: Tapentadol in an experimental animal model of acute orofacial pain. Neuro Endocrinol Lett 39: 496-500, 2019.

SIAO KT, ILKIW JE: Effects of tramadol hydrochloride on the thermal threshold in cats. Am J Vet Res 70: 1465-1470, 2009. https://doi.org/10.2460/ajvr.70.12.1465

STEIGERWALD I, MUELLER M, DAVIES A, SAMPER D, SABATOWSKI R, BARON R, ROZENBERG S, SZCZEPANSKA-SZEREJ A, GATTI A, KRESS HG: Effectiveness and safety of tapentadol prolonged release for severe, chronic low back pain with or without a neuropathic pain component: results of an openlabel, phase 3b study. Curr Med Res Opin 28: 911-936, 2012. https://doi.org/10.1185/03007995.2012.679254

TZSCHENTKE TM, CHRISTOPH T, KÖGEL B, SCHIENE K, HENNIES HH, ENGLBERGER W, HAURAND M, JAHNEL U, CREMERS TI, FRIDERICHS E, DE VRY J: J Pharmacol Exp Ther 323: 265-276, 2007. https://doi.org/10.1124/jpet.107.126052 